V.M. Boev

\title{
CALCULATION OF TRANSIENTS IN ELECTRICAL CIRCUITS WITH «INCORRECT» INITIAL CONDITIONS WITH THE HELP OF THE DUHAMEL INTEGRAL AND DISCONTINUOUS FUNCTIONS
}

A technique for calculating transients using the Duhamel integral and discontinuous functions is presented. On specific examples, the procedure for calculating "incorrect» problems with respect to differential equations, compiled according to Kirchhoff laws, and using the Duhamel integral is presented. In this case, the Kirchhoff law and the transition characteristic in the Duhamel integral are written using unitary discontinuous functions for the electrical circuit as a whole (before and after commutation). It is shown that the application of discontinuous functions for describing piecewise continuous input signals and switching in an electric circuit extends the domain of applicability of the Duhamel integral. References 9, figures 3.

Key words: transients, Duhamel integral, discontinuous functions.

Излагается методика расчета переходных процессов с использованием интеграла Дюамеля и разрывных функций. На конкретных примерах излагается порядок расчета «некорректных" задач по дифференциальным уравнениям, составляемым по законам Кирхгофа, и с помощью интеграла Дюамеля. При этом законы Кирхгофа и переходная характеристика в интеграле Дюамеля записываются с помощью единичных разрывных функций для электрической цепи в целом (до и после коммутации). Показано, что применение разрывных функций для описания кусочнонепрерывных входных сигналов и переключений в электрической цепи расииряет область применимости интеграла Дюамеля. Библ. 9, рис. 3.

Ключевые слова: переходные процессы, интеграл Дюамеля, разрывные функции.

The state of the art and problem definition. In theoretical electrical engineering, the basic methods for calculating transients in electrical circuits are: classical, operator, frequency (spectral) and based on the use of the Duhamel integral [1]. The domain of preferential application of the Duhamel integral is electrical circuits with an input signal of arbitrary shape.

In recent years, publications have appeared in which the Duhamel integral is used to calculate the process of propagation of the electromagnetic field (lightning discharges, industrial interferences, etc.) in an inhomogeneous medium $[2,3]$. In this case, the field problem is represented by a substitution circuit in the form of a long line or a four-terminal network [3, 4]. The transition characteristic required for the Duhamel integral is determined by the substitution circuit. In [5], the Duhamel integral is used in the calculation of the electromagnetic field in a layered medium. Thus, the Duhamel integral remains a sought-after method and the extension of its applicability range (in this case to electric circuits with «incorrect» initial conditions, when the switching laws in the formulation for current in the inductance and the voltage on the capacitance are not applicable) is relevant.

The drawbacks of the Duhamel integral include the requirement of zero initial conditions and the impossibility of taking into account the switching that changes the structure of the electrical circuit. These constraints can be leveled by using discontinuous (stepwise) functions to describe piecewise continuous input signals and changes in the structure of the circuit during switching.

The connection of an electric circuit at a constant voltage $U_{1}$ at zero initial conditions can be considered as an action of the input voltage $U=1(t) U_{1}$ in the circuit already switched on [1], where $1(t)$ is the Heaviside unit function (connection function) (see Fig. 1). This statement is also true for the variable input voltage $u(t)=u(t)=1(t) \cdot u_{1}(t)$. Then the Duhamel integral can be represented in the form of the integral

$$
i(t)=\int_{-0}^{t} u^{\prime}(\Theta) h(t-\Theta) d \Theta, \quad i(t)=\int_{-t_{0}}^{t} u^{\prime}(\Theta) h(t-\Theta) d \Theta,
$$

where

$$
\begin{aligned}
& u^{\prime}(\Theta)=\left.\frac{d u(t)}{d t}\right|_{t=\Theta}=\left.\left[1(t) u_{1}(t)\right]^{1}\right|_{t=\Theta}=\left[\delta(t) u_{1}(0)+\right. \\
& \left.+1(t) u^{\prime}(t)_{1}\right]\left.\right|_{t=\Theta}=\delta(\Theta) u_{1}(0)+1(\Theta) u_{1}^{\prime}(\Theta) .
\end{aligned}
$$

Then

$$
\begin{aligned}
& i(t)=\int_{-t_{0}}^{t}\left[\delta(\Theta) u_{1}(0)+1(\Theta) u_{1}^{\prime}(\Theta)\right] h(t-\Theta) d \Theta= \\
& =u_{1}(0) h(t) \int_{-t_{0}}^{t} \delta(\Theta) d \Theta+\int_{-t_{0}}^{t} u_{1}^{\prime}(\Theta) h(t-\Theta) \cdot 1(\Theta) d \Theta= \\
& =u_{1}(0) h(t)+\int_{0}^{t} u_{1}^{\prime}(\Theta) h(t-\Theta) d \Theta .
\end{aligned}
$$

Here, the filtering property of the unit function and the $\delta$-function is taken into account. $\Theta$ is the time of occurrence of voltage surges into which the input voltage $u_{1}(t)$ in accordance with the physical meaning of the Duhamel integral is divided, $(t-\Theta)$ is the time of action of each of the voltage jumps, $h(t-\Theta)$ is the transition conductivity for each of the voltage jumps.

Formula (2) is one of the varieties of the Duhamel integral. Formulas (1), (2) are written for the current. But the output function can be voltage (or current) in any branch of the electrical circuit and then the transition conductivity $h(t-\Theta)$ should be replaced by the corresponding voltage (or current) transition function.

If the input signal $u_{1}(t)$ starts to act at $t<0$, then in the formulas (1), (2) the lower limit of integration can be referred to infinity $t_{0}=\infty$. 
It was shown in $[6,7]$ that the transient occurring in an electrical circuit under the action of a complex piecewise continuous signal (including the one starting to act at $t=t_{0}<0$ ) can be calculated in two ways:

1. Description of the input signal and the general form of the solution by one analytical expression with the help of single stepwise functions and substitution of the general form of the solution in the differential equation for the sought value.

2. By the formulas of the Duhamel integral. In this case, the description of the input signal by one analytical expression with the help of discontinuous (stepwise) functions allows us to use the Duhamel integral for signals that begin to act also for $t=t_{0}<0$.

Examples of such calculations are given in $[6,7]$.

Non-zero initial conditions take place in the electrical circuit when the transient occurs as a result of a change in the structure of the circuit (connection or disconnection of individual circuit elements). The transient can also be calculated in the following two ways:

1. The change in the parameters of the electric circuit is described by means of discontinuous functions and is found in the differential equation for the sought value. The input voltage is assumed to be switched on in some preceding commutation time $t=t_{0}<0$. We write the solution of the differential equation with the aid of discontinuous functions as consisting of two parts (for $t<0$ and $t>0$ ) and substitute it into the differential equation. If the process is assumed to be steady before the commutation $(t=0)$, then this will be the initial state of the circuit with non-zero initial conditions (in this case, only the forced component is used in the solution for $t<0)$.

2. In the formulas of the Duhamel integral, the input voltage is also considered to begin to act at the time $t=t_{0}$ $<0$ which is written using discontinuous functions. The transient function $h(t)$ (with respect to current or voltage) is written using discontinuous functions as consisting of two parts, corresponding to the circuits before and after commutation.

In $[6,7]$, examples are given with switching in circuits that change the active resistance $R$. The question of transients for the general case, with switching changes in the inductance $L$ and capacitance $C$ (when the switching laws in the formulation for the current in the inductance and the voltage in the capacitor not applicable) remains unresolved, which is the subject of this paper.

The goal of the paper is to justify the possibility of calculating transients in an electrical circuit with «incorrect» initial conditions using the Duhamel integral and discontinuous functions.

Main part. To describe the sudden changes in the voltages, currents and parameters of the electrical circuit, we use discontinuous functions written with the aid of the modulo function [6] (Fig. 1):

- Fig. 1,a: $f_{1}(t)=1(t-a)=\frac{1}{2}\left(1+\frac{|t-a|}{t-a}\right)-$ unit Heaviside function;
- Fig. 1,b:

$$
\begin{aligned}
& f_{2}(t)=1(t-a)+1(b-t)-1=\frac{1}{2}\left(\frac{|t-a|}{t-a}-\frac{|t-b|}{t-b}\right), \quad a<b ; \\
& \text { - Fig. 1,c: } f_{3}(t)=1(b-t)=\frac{1}{2}\left(1-\frac{(t-b)}{t-b}\right)-\text { inverse }
\end{aligned}
$$

Heaviside function

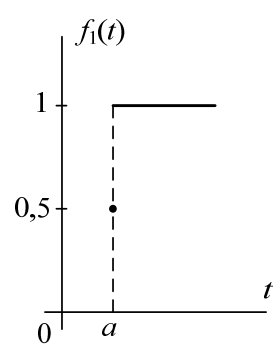

$a$

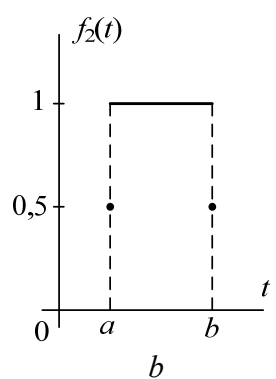

Fig. 1

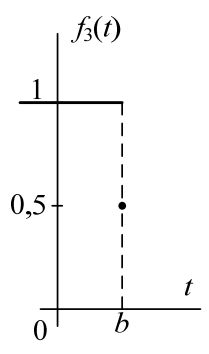

$c$
We also calculate the differential equations, compiled according to Kirchhoff laws, and the Duhamel integral. In the first case, Kirchhoff laws are compiled for the electrical circuit as a whole (before and after commutation), and the difference of these circuits is taken into account for by unitary discontinuous functions.

In the second case, the transition characteristic in the Duhamel integral is written for the circuit as a whole (before and after commutation) by means of unit discontinuous functions.

We consider the circuit (Fig. 2), in which the current in the inductance changes abruptly.

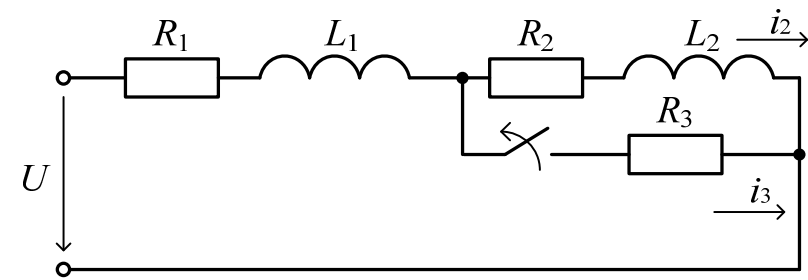

Fig. 2

Kirchhoff laws for such a circuit:

$$
\begin{gathered}
R_{1} i_{1}+L_{1} \frac{d i_{1}}{d t}+R_{2} i_{2}+L_{2} \frac{d i_{2}}{d t}=U ; \\
R_{2} i_{2}+L_{2} \frac{d i_{2}}{d t}=R_{3} i_{3} ; \\
i_{1}=i_{2}+i_{3} \frac{1}{2}\left(1-\frac{|t|}{t}\right) .
\end{gathered}
$$

Here we took into account the change in the structure of the parallel section with the help of single discontinuous functions, and written Kirchhoff laws for the circuit as a whole (before and after switching).

From (4) we find

$$
i_{3}=\frac{R_{2}}{R_{3}} i_{2}+\frac{L_{2}}{R_{3}} \frac{d i_{2}}{d t} .
$$

We solve the problem with respect to the current $i_{2}$ : 


$$
i_{2}(t)=\frac{1}{2}\left(1-\frac{|t|}{t}\right) i_{0}(t)+\frac{1}{2}\left(1+\frac{|t|}{t}\right) i(t) .
$$

Then from equation (5) we obtain:

$$
\begin{aligned}
& i_{1}(t)=\frac{1}{2}\left(1-\frac{|t|}{t}\right)\left(i_{0}+i_{3}\right)+\frac{1}{2}\left(1+\frac{|t|}{t}\right) i= \\
& =\frac{1}{2}\left(1-\frac{|t|}{t}\right)\left(i_{0}+\frac{R_{2}}{R_{3}} i_{0}+\frac{L_{2}}{R_{3}} \frac{d i_{0}}{d t}\right)+\frac{1}{2}\left(1+\frac{|t|}{t}\right) i .
\end{aligned}
$$

We substitute this in equation (3):

$\frac{1}{2}\left(1-\frac{|t|}{t}\right)\left[\left(1+\frac{R_{2}}{R_{3}}\right) R_{1} i_{0+} \frac{R_{1}}{R_{3}} L_{2} \frac{d i_{0}}{d t}\right]+L_{1}\left[\left(1+\frac{R_{2}}{R_{3}}\right) \frac{d i_{0}}{d t}+\right.$ $\left.+\frac{L_{2}}{R_{3}} \frac{d^{2} i_{0}}{d t^{2}}\right] \frac{1}{2}\left(1-\frac{|t|}{t}\right)+L_{1}\left[\left(1+\frac{R_{2}}{R_{3}}\right) i_{0}+\frac{L_{2}}{R_{3}} \frac{d i_{0}}{d t}\right](-\delta(t))+$

$+\frac{1}{2}\left(1-\frac{|t|}{t}\right)\left[R_{2} i_{0}+L_{2} \frac{d i_{0}}{d t}\right]-\delta(t) i_{0} L_{2}+\frac{1}{2}\left(1+\frac{|t|}{t}\right) \times$

$\times\left[\left(R_{1}+R_{2}\right) i+\left(L_{1}+L_{2}\right) \frac{d i}{d t}\right]+\delta(t)\left(L_{1}+L_{2}\right) i=$

$=U=\frac{1}{2}\left(1-\frac{|t|}{t}\right) U+\frac{1}{2}\left(1+\frac{|t|}{t}\right) U$.

We equate multipliers for the same discontinuous functions:

1) $\frac{1}{2}\left(1-\frac{|t|}{t}\right): \frac{R_{3}+R_{2}}{R_{3}}\left(R_{1} i_{0}+\frac{d i_{0}}{d t} L_{1}\right)+$

$+\frac{L_{2}}{R_{3}}\left(R_{1} \frac{d i_{0}}{d t}+L_{1} \frac{d^{2} i_{0}}{d t^{2}}\right)+R_{2} i_{0}+L_{2} \frac{d i_{0}}{d t}=U$,

2) $\frac{1}{2}\left(1+\frac{|t|}{t}\right):\left(R_{1}+R_{2}\right) i+\left(L_{1}+L_{2}\right) \frac{d i}{d t}=U$,

3) $\delta(t): L_{1} \frac{R_{3}+R_{2}}{R_{3}} i_{0}+\frac{L_{1} L_{2}}{R_{3}} \cdot \frac{d i_{0}}{d t}+L_{2} i_{0}=\left(L_{1}+L_{2}\right) i$.

Equation (6) is the differential equation of the circuit before commutation:

$$
\begin{aligned}
& \frac{L_{1} L_{2}}{R_{3}} \frac{d^{2} i_{0}}{d t^{2}}+\left(\frac{R_{1}}{R_{3}} L_{2}+\frac{R_{3}+R_{2}}{R_{3}} L_{1}+L_{2}\right) \frac{d i_{0}}{d t}+ \\
& +\frac{R_{3}+R_{2}}{R_{3}} R_{1} i_{0}+R_{2} i_{0}=U .
\end{aligned}
$$

Its solution:

$$
i_{0}(t)=i_{\text {force }}+i_{\text {free }}=i_{\text {force }}+A_{1} e^{k_{1} t}+A_{2} e^{k_{2} t},
$$

where $i_{\text {force, }} i_{\text {free }}$ are the forced and free current components respectively.

But before commutation, we are interested in the steady-state process, i.e. $i_{\text {force }}=$ const :

$$
i_{\text {force }}=i_{0}=\frac{U R_{3}}{\left(R_{2}+R_{3}\right) R_{1}+R_{2} R_{3}}=i_{2}(0-) \text {. }
$$

Equation (7) is the differential equation of the circuit after commutation. Its solution:

$$
i(t)=i_{\text {force }}+i_{\text {free }}=\frac{U}{R_{1}+R_{2}}+A e^{-\frac{t}{\tau}} ; \quad \tau=\frac{L_{1}+L_{2}}{R_{1}+R_{2}} .
$$

In equation (8) we take into account that $d i_{0} / d t=0$, since $i_{0}=i_{\text {force }}=$ const and $\frac{R_{3}+R_{2}}{R_{3}} i_{0}=i_{1}(0-)$. Then

$$
L_{1} i_{1}(0-)+L_{2} i_{2}(0-)=\left(L_{1}+L_{2}\right) i_{2}(0+),
$$

since in our notation $i(0)=i_{2}(0+)$.

Thus, equation (8) is the first commutation law for flux linkages.

We substitute values:

$$
\begin{aligned}
& \left(\frac{R_{2}+R_{3}}{R_{3}} L_{1}+L_{2}\right) \frac{U R_{3}}{R_{1}\left(R_{2}+R_{3}\right)+R_{2} R_{3}}= \\
& =\left(\frac{U}{R_{1}+R_{2}}+A\right)\left(L_{1}+L_{2}\right) .
\end{aligned}
$$

We found the constant $A$ :

$$
\begin{aligned}
& A=\frac{U}{L_{1}+L_{2}} \cdot \frac{\left(R_{2}+R_{3}\right) L_{1}+R_{3} L_{2}}{R_{1}\left(R_{2}+R_{3}\right)+R_{2} R_{3}}-\frac{U}{R_{1}+R_{2}}= \\
& =\frac{U R_{2}\left(L_{1} R_{2}-L_{2} R_{1}\right)}{\left(L_{1}+L_{2}\right)\left(R_{1}+R_{2}\right)\left(R_{1} R_{2}+R_{1} R_{3}+R_{2} R_{3}\right)} .
\end{aligned}
$$

The same solution was obtained in [8].

If $R_{3}=0$, i.e. before switching the section $\left(R_{2}-L_{2}\right)$ was short-circuited, then

$$
A=\frac{U\left(L_{1} R_{2}-L_{2} R_{1}\right)}{\left(L_{1}+L_{2}\right)\left(R_{1}+R_{2}\right) R_{1}},
$$

which coincides with the solution given in [1].

We solve this problem using the Duhamel integral and obtain the same result. We believe that the electrical circuit before switching was switched on to the voltage $U$ at the time $t=-t_{0}<0$

$$
u(t)=\frac{1}{2}\left(1+\frac{\left|t+t_{0}\right|}{t+t_{0}}\right) U .
$$

Assuming that the transient from the switching on to the time of commutation $t=0$ has already ended, we write the transient conductivity of the circuit before commutation for the current $i_{2}$ by the forced component (9):

$$
h_{0}(t)=\frac{R_{3}}{R_{1} R_{2}+R_{1} R_{3}+R_{2} R_{3}} .
$$

The transient conductivity for the circuit after commutation, according to (10), (11), is equal to:

$$
h(t)=\frac{1}{R_{1}+R_{2}}+\frac{R_{2}\left(L_{1} R_{2}-L_{2} R_{1}\right)}{\left(L_{1}+L_{2}\right)\left(R_{1}+R_{2}\right)\left(R_{1} R_{2}+R_{1} R_{3}+R_{2} R_{3}\right)} e^{-\frac{t}{\tau}} .
$$

Then, according to (1), we obtain:

$$
\begin{gathered}
i_{2}(t)=\int_{-0}^{t} u^{\prime}\left(\Theta^{\prime}\right)\left[\frac{1}{2}\left(1-\frac{|t|}{t}\right) h_{0}\left(t-\Theta^{\prime}\right)+\frac{1}{2}\left(1+\frac{|t|}{t}\right) h\left(t-\Theta^{\prime}\right)\right] d \Theta^{\prime}= \\
=\int_{-t_{0}}^{t} U \delta\left(\Theta+t_{0}\right)\left[\frac{1}{2}\left(1-\frac{|t|}{t}\right) \frac{R_{3}}{R_{1} R_{2}+R_{1} R_{3}+R_{2} R_{3}}+\frac{1}{2}\left(1+\frac{|t|}{t}\right) \times\right. \\
\times\left[\frac{1}{R_{1}+R_{2}}+\frac{R_{2}\left(L_{1} R_{2}-L_{2} R_{1}\right)}{\left(L_{1}+L_{2}\right)\left(R_{1}+R_{2}\right)\left(R_{1} R_{2}+R_{1} R_{3}+R_{2} R_{3}\right)} \times\right. \\
\times e^{\left.\left.-\frac{\left(t-\Theta-t_{0}\right)}{\tau}\right]\right] d \Theta=\frac{1}{2}\left(1-\frac{|t|}{t}\right) \frac{R_{3} U}{R_{1} R_{2}+R_{1} R_{3}+R_{2} R_{3}}+}
\end{gathered}
$$




$$
\begin{gathered}
+\frac{1}{2}\left(1+\frac{|t|}{t}\right)\left[\frac{U}{R_{1}+R_{2}}+\right. \\
+\frac{R_{2}\left(L_{1} R_{2}-L_{2} R_{1}\right) U}{\left(R_{1} R_{2}+R_{1} R_{3}+R_{2} R_{3}\right)\left(L_{1}+L_{2}\right)\left(R_{1}+R_{2}\right)} e^{-\frac{t}{\tau}} .
\end{gathered}
$$

Here: $\Theta^{\prime}=\Theta+t_{0}$ is the input signal coordinate

$$
\int_{-t_{0}^{\prime}}^{t} \delta\left(\Theta+t_{0}\right) d \Theta=1 ; \int_{-t_{0}^{\prime}}^{t} \delta\left(\Theta+t_{0}\right) f d \Theta=f\left(-t_{0}\right) .
$$

Here: the lower limit $-t_{0}^{\prime}$ is less than $-t_{0}$ by an infinitesimal value (i.e. $-t_{0}^{\prime}=-t_{0}-0$ );

$$
\frac{1}{2}\left(1-\frac{|t|}{t}\right)=1 \quad(\text { at } t<0) \text { is the current multiplier }
$$
before commutation;

$$
\left.\frac{1}{2}\left(1+\frac{|t|}{t}\right)=1 \quad \text { at } t>0\right) \text { is the current multiplier }
$$

before commutation;

These multipliers do not take part in integration with respect to $\Theta$, since they separate the range of the formulas of the transient conductivity of the output signal before and after switching.

We consider a circuit with a capacitive energy storage device, where commutation changes the capacitance in the circuit (Fig. 3).

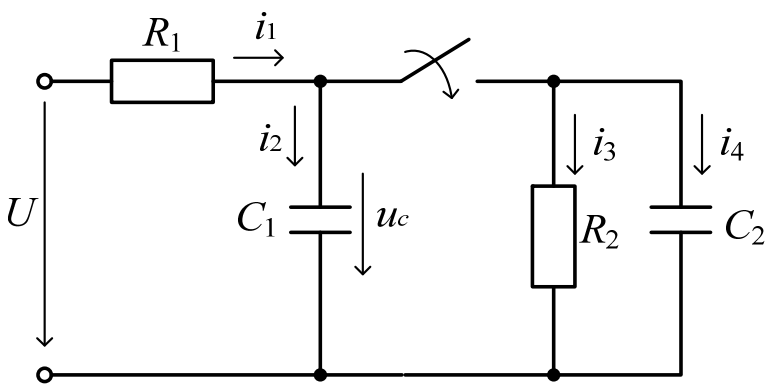

Fig. 3

Initial data: $U=60 \mathrm{~V}, R_{1}=R_{2}=1 \mathrm{k} \Omega$, $C_{1}=1 \mu \mathrm{F}, C_{2}=2 \mu \mathrm{F}$. We determine $i_{1}$

Kirchhoff laws for the circuit as a whole (before and after switching):

$$
\begin{gathered}
R_{1} i_{1}+u_{c}=U ; \\
i_{1}=i_{2}+\frac{1}{2}\left(1+\frac{|t|}{t}\right)\left(i_{3}+i_{4}\right) ; \\
i_{2}=c_{1} \frac{d u_{c}}{d t} \\
i_{3}=\frac{u_{c}}{R_{2}} \\
i_{4}=c_{2} \frac{d u_{c}}{d t} .
\end{gathered}
$$

We substitute the currents into the first equation:

$$
R_{1} c_{1} \frac{d u_{c}}{d t}+u_{c}+\frac{1}{2}\left(1+\frac{|t|}{t}\right)\left(R_{1} c_{2} \frac{d u_{c}}{d t}+\frac{R_{1}}{R_{2}} u_{c}\right)=U \text {. }
$$

We write the voltage $u_{c}$ as the sum of the voltages before and after the commutation separated by unit discontinuous functions.

$$
u_{c}(t)=\frac{1}{2}\left(1-\frac{|t|}{t}\right) u_{0}+\frac{1}{2}\left(1+\frac{|t|}{t}\right) u \text {. }
$$

We substitute into the differential equation (13)

$$
\begin{gathered}
\frac{1}{2}\left(1-\frac{|t|}{t}\right)\left[u_{0}+R_{1} c_{1} \frac{d u_{0}}{d t}\right]-\delta(t) R_{1} c_{1} u_{0}+ \\
+\frac{1}{2}\left(1+\frac{|t|}{t}\right)\left[\left(1+\frac{R_{1}}{R_{2}}\right) u+R_{1}\left(c_{1}+c_{2}\right) \frac{d u}{d t}\right]+ \\
+\delta(t) R_{1}\left(c_{1}+c_{2}\right) u=U .
\end{gathered}
$$

We equate multipliers for the same discontinuous functions:

1) $\quad \frac{1}{2}\left(1-\frac{|t|}{t}\right): R_{1} c_{1} \frac{d u_{0}}{d t}+u_{0}=U$;

2) $\frac{1}{2}\left(1+\frac{|t|}{t}\right): R_{1}\left(c_{1}+c_{2}\right) \frac{d u}{d t}+\left(1+\frac{R_{1}}{R_{2}}\right) u=U$;

3) $\delta(t):-R_{1} c_{1} u_{0}+R_{1}\left(c_{1}+c_{2}\right) u=0$.

The solution of equation (14) for the circuit before commutation has the form:

$$
u_{0}(t)=U+A e^{-\frac{t}{\tau}}=U-U e^{\frac{-\left(t+t_{0}\right)}{\tau}} ; \quad \tau=\frac{1}{R_{1} c_{1}} .
$$

Since before the commutation at some time $t=-t_{0}<0$ the circuit $\left(R_{1}-c_{1}\right)$ was switched on to the voltage $U$, then this transient is considered to be completed before the time $t=0$. Then:

$$
u_{0}(t)=u_{c_{1}}(0-)=U \text {. }
$$

The solution of equation (15) for the circuit after commutation is:

$$
\begin{gathered}
u_{0}(t)=\frac{U R_{2}}{R_{2}+R_{1}}+A e^{\frac{-t}{\tau}}=30+A e^{\frac{-t}{\tau}} ; \\
\tau=\left(c_{1}+c_{2}\right) \frac{R_{1} R_{2}}{R_{1}+R_{2}}=1,5 \cdot 10^{-3} \mathrm{c} .
\end{gathered}
$$

Equation (16) is the second commutation law for charges

$$
c_{1} u_{0}(0-)=\left(c_{1}+c_{2}\right) u(0+),
$$

i.e.

$$
c_{1} u_{c_{1}}(0-)=\left(c_{1}+c_{2}\right) u_{c}(0+) .
$$

After substitution, we obtain equality for the determination of the constant $A$

$$
c_{1} U=\left(c_{1}+c_{2}\right)\left[\frac{U R_{2}}{R_{1}+R_{2}}+A\right] \text {. }
$$

From here:

$$
A=U\left(\frac{c_{1}}{c_{1}+c_{2}}-\frac{R_{2}}{R_{1}+R_{2}}\right)=60\left(\frac{1}{3}-\frac{1}{2}\right)=-10 .
$$

Then:

$u_{c}(t)=\frac{1}{2}\left(1-\frac{|t|}{t}\right) 60+\frac{1}{2}\left(1+\frac{|t|}{t}\right)\left(30-10 e^{-\frac{2}{3} \cdot 10^{3} t}\right)$. 
The source current:

$$
i_{1}(t)=\left(c_{1}+c_{2}\right) \frac{d u_{c}}{d t}+\frac{u_{c}}{R_{2}}=3 \cdot 10^{-2}+10^{-2} e^{-\frac{2}{3} \cdot 10^{3} t} .
$$

The same result was obtained in [9].

We solve the same problem with the help of the Duhamel integral. The transient response of the circuit for the voltage before and after commutation has the form:

$$
\begin{aligned}
h(t) & =\frac{1}{2}\left(1-\frac{|t|}{t}\right) \cdot 1+\frac{1}{2}\left(1+\frac{|t|}{t}\right)\left[\frac{R_{2}}{R_{1}+R_{2}}+\right. \\
& +\left(\frac{c_{1}}{c_{1}+c_{2}}-\frac{R_{2}}{R_{1}+R_{2}}\right) e^{\left.\frac{-\left(t-\Theta^{\prime}\right)}{\tau}\right] .}
\end{aligned}
$$

We assume that the circuit was switched on at a certain time $t=-t_{0}<0$ by the voltage (12) before the commutation, and this transient ended before the commutation time $t=0$. Then the Duhamel integral (1) has the form:

$$
\begin{aligned}
u_{c}(t)= & \int_{-0}^{t} u^{\prime}\left(\Theta^{\prime}\right) h\left(t-\Theta^{\prime}\right) d \Theta^{\prime}=\int_{-t_{0}}^{t} U \delta\left(\Theta+t_{0}\right)\left[\frac{1}{2}\left(1-\frac{|t|}{t}\right)+\right. \\
& +\frac{1}{2}\left(1+\frac{|t|}{t}\right)\left[\frac{R_{2}}{R_{1}+R_{2}}+\left(\frac{c_{1}}{c_{1}+c_{2}}-\frac{R_{2}}{R_{1}+R_{2}}\right) \times\right. \\
& \times e^{\left.\left.\frac{-\left(t-\Theta-t_{0}\right)}{\tau}\right]\right] d \Theta=\frac{1}{2}\left(1-\frac{|t|}{t}\right) U+\frac{1}{2}\left(1+\frac{|t|}{t}\right) \times} \\
& \times\left[\frac{R_{2} U}{R_{1}+R_{2}}+U\left(\frac{c_{1}}{c_{1}+c_{2}}-\frac{R_{2}}{R_{1}+R_{2}}\right) e^{\frac{-t}{\tau}}\right] .
\end{aligned}
$$

The same solution for $u_{c}$ was obtained above. If in this solution $R_{2} \rightarrow \infty$, then we obtain a solution for the circuit (Fig. 3) without $R_{2}$, which is given in [1]:

$$
u_{c}(t)=U+U\left(\frac{c_{1}}{c_{1}+c_{2}}-1\right) e^{\frac{-t}{\tau}} \text {. }
$$

Conclusions.

1. For the first time, the possibility of calculating transients in an electric circuit with «incorrect» initial conditions with the help of the Duhamel integral and discontinuous functions is justified.
2. The proposed solution of the problem of calculating transients in the electrical circuit with non-zero and «incorrect» initial conditions with the help of the Duhamel integral is more compact than the known ones.

\section{REFERENCES}

1. Neyman L.R., Demirchyan K.S. Teoreticheskie osnovy elektrotekhniki. V 2-kh t. T. 1 [Theoretical bases of electrical engineering. In 2 vols. Vol. 1]. Leningrad, Energoizdat Publ., 1981, p. 536. (Rus).

2. Kochetov S.V., Wollenberg G. Stable and Effective FullWave PEEC Models by Full-Spectrum Convolution Macromodeling. IEEE Transactions on Electromagnetic Compatibility, 2007, vol.49, no.1, pp. 25-34. doi: 10.1109/temc.2006.888183.

3. Konnikov I.A. Interference of an elementary source of an electromagnetic field in an electronic module. Technology of electromagnetic compatibility, 2006, no.4, pp.18-26. (Rus).

4. Elmore W.C. The Transient response of Damped Linear Networks with Particular Regard to wideband Amplifiers. Journal of Applied Physics, 1948, vol.19, no.1, pp. 55-63. doi: 10.1063/1.1697872.

5. Konnikov I.A. Calculation of the electromagnetic field in a layered medium. Electricity, 2017, no.7, pp. 60-67. (Rus).

6. Boev V.M. The use of discontinuous functions for the calculation of transient processes and impulse actions in linear electric circuits. 1. Transient processes. Electronic modeling, 2002, vol.24, no.6, pp. 67-79. (Rus).

7. Boev V.M. The use of discontinuous functions for the calculation of transient processes and impulse actions in linear electric circuits. 2. Impulse effects. Electronic modeling, 2003, vol.25, no.1, pp. 83-97. (Rus).

8. Rybalko M.P., Esaulenko V.O., Kostenko V.I. Teoretichni osnovi elektrotehniki. Liniyni elektrichni kola: Pidruchnik. [Theoretical foundations of electrical engineering. Linear electric circuits: Textbook]. Donetsk, Novyi Svit Publ., 2003. 513 p. (Ukr).

9. Shebes M.P. Zadachnik po teorii lineynyih elektricheskih tsepey [Tasks of problems in the theory of linear electrical circuits]. Moscow, Vysshaya Shkola Publ., 1982. 488 p. (Rus).

Received 03.04.2018

V.M. Boev, Doctor of Technical Science, Professor,

National Technical University «Kharkiv Polytechnic Institute», 2, Kyrpychova Str., Kharkiv, 61002, Ukraine, phone +380 577076961

\section{How to cite this article:}

Boev V.M. Calculation of transients in electrical circuits with «incorrect» initial conditions with the help of the Duhamel integral and discontinuous functions. Electrical engineering \& electromechanics, 2018, no.4, pp. 40-44. doi: 10.20998/2074-272X.2018.4.07. 